\title{
Studying Electron Transfer Pathways in Oxidoreductases
}

\author{
María Gabriela Rivas, Pablo J. González, Felix M. Ferroni, Alberto C. Rizzi, and \\ Carlos D. Brondino*
}

Departamento de Física, Facultad de Bioquímica y Ciencias Biológicas, Universidad Nacional del Litoral, Santa Fe, Argentina. ${ }^{*}$ Corresponding author. E-mail: brondino@fbcb.unl.edu.ar

\begin{abstract}
Oxidoreductases containing transition metal ions are widespread in nature and are essential for living organisms. The copper-containing nitrite reductase (NirK) and the molybdenum-containing aldehyde oxidoreductase (Aor) are typical examples of oxidoreductases. Metal ions in these enzymes are present either as mononuclear centers or organized into clusters and accomplish two main roles. One of them is to be the active site where the substrate is converted into product, and the other one is to serve as electron

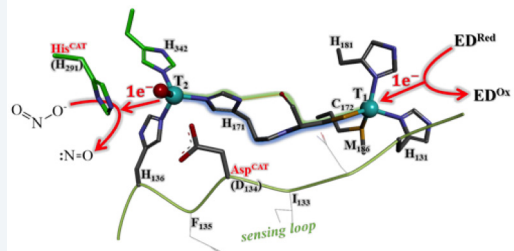
transfer center. Both enzymes transiently bind the substrate and an external electron donor/acceptor in NirK/Aor, respectively, at distinct protein points for them to exchange the electrons involved in the redox reaction. Electron exchange occurs through a specific intra-protein chemical pathway that connects the different enzyme metal cofactors. Based on the two oxidoreductases presented here, we describe how the different actors involved in the intra-protein electron transfer process can be characterized and studied employing molecular biology, spectroscopic, electrochemical, and structural techniques.
\end{abstract}

\section{Keywords:}

electron transfer pathways, oxidoreductases, transition metal ions

\section{Introduction}

Proteins are the most versatile macromolecules in living organisms. They have key roles in every metabolic pathway occurring inside living cells as (bio)catalysts, transporting and/or storing essential molecules, providing mechanical support and immune protection, generating movement, transmitting electric impulses, and controlling cell growth and differentiation (Berg et al., 2012). About a third of all proteins present in living organisms contains one or more transition metal ions as cofactors and are called metalloproteins or metalloenzymes, accordingly (Silva and Williams, 1991). Metalloenzymes occur in the six Enzyme Commission Classes (E.C.C.). Within the oxidoreductases group (EC 1), almost half (44\%) are metalloenzymes. A large percentage of transferases (EC 2, 40\%), hydrolases (EC 3, 39\%), lyases (EC 4, $36 \%$ ), isomerases (EC 5, 36\%), and ligases (EC 6, 59\%), are also metalloenzymes (Andreini et al., 2008).

Metalloenzymes of the oxidoreductase group catalyze specific reactions in which metal ions are part of the enzyme active site, function as electron transfer (ET) centers, or, less frequently, stabilize protein structure. These enzymes incorporate $81 \%$ of the total Fe found in living organisms, as well as $93 \%$ of the total $\mathrm{Cu}$, and $81 \%$ of total Mo plus $\mathrm{W}$ (Andreini et al. 2008). These four metals are the so-called redox-active elements of life since they can exist in several 
oxidation states within the physiological range of electrochemical potential that can be handled by a living cell ( $c a$ from -800 to $+800 \mathrm{mV}, v s \mathrm{SHE}$ ). The redox cycling of these transition metal ions is the basis for them to work as electron conduits, mediating ET from the enzyme active site to a redox partner, and vice versa.

Oxidoreductases have an active site where the oxidation or reduction of the substrate occurs. In the first case, electrons obtained from substrate oxidation reduce the active site, and then are transferred through redox cofactors buried in the protein to a surface exposed center capable of reducing an electron acceptor (EA). On the other hand, when the enzyme catalyzes the reduction of a substrate, the process is the inverse. The electron donor (ED) transfers electrons to a surface exposed redox center of the enzyme, and electrons are conducted through an intra-protein chemical pathway to the active site where substrate reduction occurs. The processes described above stand for an important group of oxidoreductases, even though some develop the redox reaction in a single protein site. It should be noted that oxidoreductases catalyze bisubstrate reactions and the labeling of substrate and ED (or EA) is biased by the interests of the researcher. For example, a compound can be called "the substrate" because it is economically relevant for the pharma, fuel, or fine chemicals industry, or simply because it reacts at a particular enzyme site that is being thoroughly studied because it has some relevant and/or novel properties. Even though we will use this jargon throughout the manuscript, it is important to remark that the correct way of stating this is that an oxidoreductase has two substrates.

The oxidoreductases we describe here perform long-distance ET reactions. Long-range ET, which occurs through electron tunneling either within an enzyme or between proteins, is fundamental to several cellular processes, mainly those related to energy metabolism (Davidson, 1996; Davidson, 2002). Therefore, for researchers working with redox enzymes and ET proteins, the knowledge of ET theory and the role of the parameters that control ET rates are relevant to understand at a molecular level how an enzyme regulates a physiological process. Moreover, this knowledge might be used to design biocatalysts by exploiting the chemistry of oxidoreductases, or even to understand the effect of a point mutation in human diseases (Moser et al., 2010).

The aim of this paper is to describe different experimental techniques that can be used to characterize and understand ET processes in oxidoreductases, and how some parameters that control ET rates can be experimentally measured or at least estimated independently. First, we will explain how to use voltamperometric and spectropotentiometric methods to characterize the redox properties of the metal centers comprising the ET pathway. Then, we will discuss different strategies to evaluate the ability of a given chemical path to act as ET pathway. Finally, we will show how X-ray data and molecular biology techniques, more specifically site-directed mutagenesis, can be used to predict ET pathways within a protein. All these methodologies/strategies will be explained based on two representative examples of oxidoreductases $-\mathrm{a}$ copper-containing nitrite reductase (NirK), which catalyzes the reduction of nitrite to nitric oxide, and a molybdenumcontaining aldehyde oxidoreductase (Aor), which catalyzes the oxidation of aldehyde to the respective carboxylic acid.

\section{Structural aspects and general reaction mechanisms of NirK and Aor}

Figure 1 shows the essential metal cofactors for catalysis in NirK and Aor together with the intra-protein chemical pathways that link the metal cofactors through which ET occurs. Copper-containing NirK presents a homotrimeric structure with two copper atoms per monomer $\sim 12 \AA$ apart, one of type 1 (T1, also blue copper) and the other of type 2 (T2, also normal copper) (Nojiri, 2017). T1 and T2 are the ET center and the active site, respectively. The T1 copper ion is tetracoordinated with three strongly bound ligands, two $\mathrm{N}$ atoms from histidine imidazoles and a cysteine thiolate group, and a weaker methionine thioether group. Subtle differences in the T1 electronic structure determines the green or blue color of NirK from different organisms. On the other hand, T2 consists of a four-coordinate copper site bound to a labile water molecule and three $\mathrm{N}$ atoms from histidine imidazoles in a distorted tetrahedral geometry. The two copper sites are connected by two main chemical pathways - the shorter one, the Cys-His bridge, is thought to transport the electron needed for nitrite reduction to nitric oxide; the longer pathway, which has been called the substrate-sensing loop, is thought to work as a relay to trigger the $\mathrm{T} 1 \rightarrow \mathrm{T} 2$ electron flow through the Cys-His bridge upon nitrite-T2 binding (Strange et al., 1999; Boulanger et al., 2000). This sensing loop contains an aspartic acid residue (Asp ${ }^{\mathrm{CAT}}$ ) that forms a hydrogen bond with the T2 labile water molecule in the resting state, and with the nitrite-T2 complex in NirK reacted with nitrite (not shown); Asp ${ }^{\mathrm{CAT}}$ was proposed to be essential in catalysis (Boulanger et al., 2000, Kataoka et al., 2000). The proposed reaction mechanism for NirK implies a two-proton coupled redox reaction in which nitrite, after binding T2, is converted to nitric oxide by one electron delivered by an external physiological ED (Suzuki et al., 1994; Fukuda et al., 2016; Nojiri 2017). The physiological ED of NirK were identified to be pseudoazurin, azurin or cytochrome $c$, depending on the microorganism. Thus, the complete ET implies three intermolecular reactions: an inter-protein ET between the physiological ED and the NirK T1 center, the T1-T2 intra-protein ET, and the ET reaction between T2 and substrate. Hereafter, the ET pathways discussed in this paper will refer only to the protein intramolecular step. 


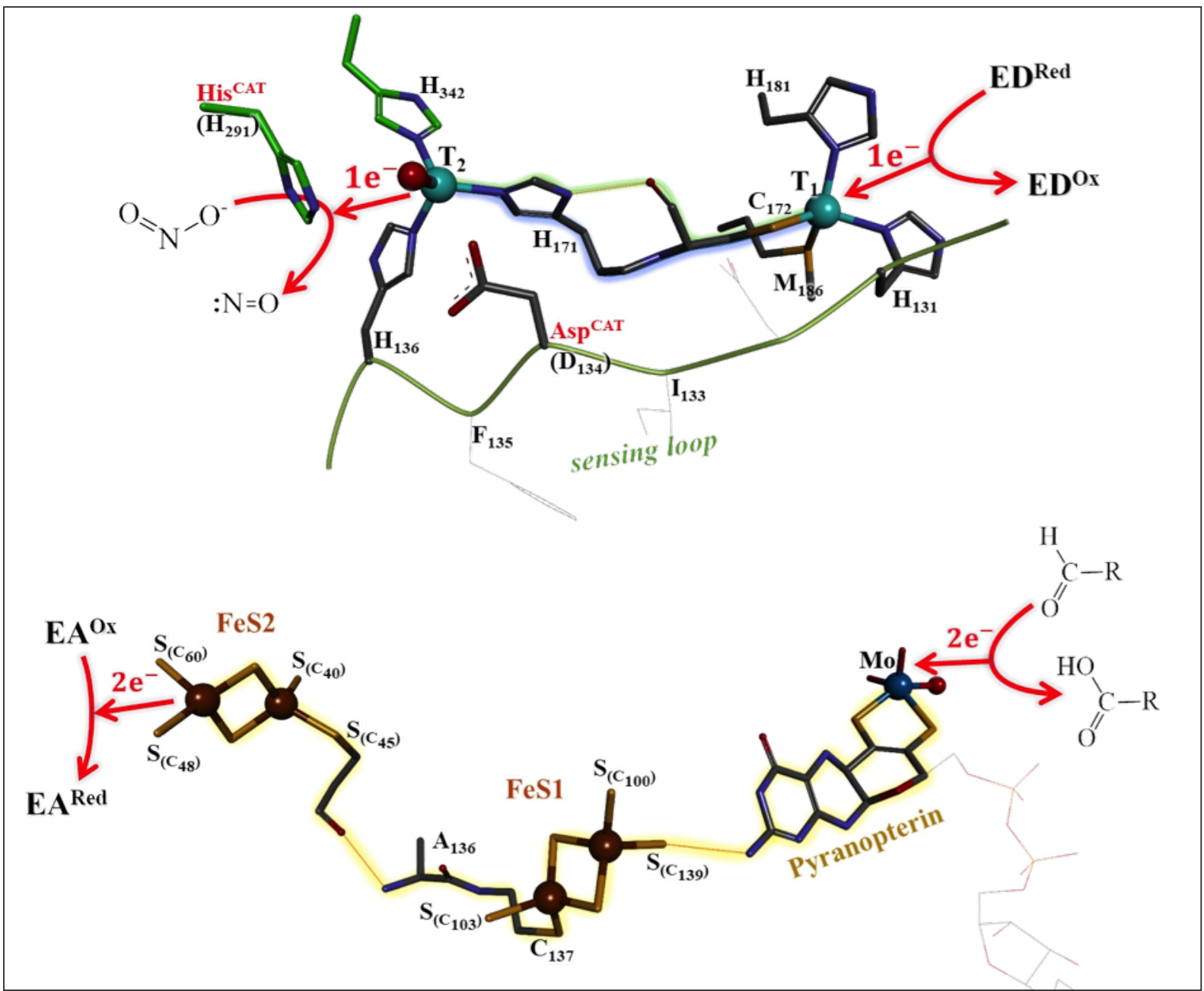

Figure 1. Metal centers in NirK (top) and Aor (bottom) together with the proposed intra-protein ET pathways. For NirK, the two alternative intra-protein ET pathways are highlighted in blue and green (Hadt et al., 2014). The blue subpath is a pure covalent chemical pathway constituted by the protein backbone and the H171 and C172 side chains, whereas the green one involves the His- $\mathrm{N}^{\delta 1} \mathrm{H} . . \mathrm{O}=\mathrm{C}$-Cys hydrogen bond that partially shortcuts the covalent link. For Aor, the proposed intra-protein ET pathway is highlighted in yellow. Structural drawings were performed with PDB structures 1SNR using Sinorhizobium meliloti NirK numbering, and 1VLB for Aor using Desulfovibrio gigas Aor numbering. The labels FeS1 and FeS2 in Aor correspond to the proximal and distal FeS centers, respectively, relative to the Mo atom position.

Aor is an oxidoreductase with more complex metal cofactors than those of NirK. Aor contains different types of metal ions at the active site and ET centers. The active site, where aldehydes are oxidized, comprises a $\mathrm{Mo}^{6+}$ ion coordinated to two oxo, one hydroxo/water $\left(\mathrm{OH}_{\mathrm{x}}\right)$ and two sulfur ligands, the latter provided from a dithiolene moiety which is part of a pyranopterin cytidine dinucleotide (Figure 1, bottom). This complex molecule, together with two iron-sulfur (FeS) clusters of the [2Fe-2S] type, are the redox centers of Aor and comprise the ET pathway (Romao et al., 1995; Rebelo et al., 2001). The FeS1 (or proximal center) is closer to the Mo site $\left(\mathrm{d}_{\text {Mo-FesI }}=16.2 \AA\right)$ and is buried inside the protein in a domain inaccessible to solvent. The FeS2 (or distal center) is further away from the Mo site $\left(\mathrm{d}_{\text {Mo-Fes2 }}=25.6 \AA\right.$ ) and close to the protein surface, and it is able to transfer electrons to a flavodoxin, the physiological electron acceptor (Brondino et al. 2006, Krippahl et al. 2006). Upon aldehyde oxidation at the active site, $\mathrm{Mo}^{6+}$ is reduced to $\mathrm{Mo}^{4+}$. The two reducing equivalents are sequentially transferred through the FeS centers to flavodoxin, as shown in Figure 1.

\section{A brief description of ET theory}

Most biological reactions, mainly those related to cell energy, involve long-range intra- and inter-protein ET, sometimes coupled to proton transfer (Formosinho and Barroso, 2012), between redox centers. A description of ET processes 
occurring both in proteins and in small molecules can be made on the basis of Marcus theory (Marcus and Sutin, 1985). The study is relatively straightforward for small molecules. However, modelling ET processes in oxidoreductases, and in all ET proteins, is complicated as it requires to understand all the phenomena that control ET in a protein, e.g. protein dynamics, which may include non-ET processes that tune ET rates (Moser et al., 2010). The Marcus model (equation 1) states that the ET rate $\left(k_{\mathrm{ET}}\right)$ is governed by the thermodynamic driving force $\left(\Delta G^{\mathrm{o}}\right)$, the reorganization energy $(\lambda)$, and the electronic coupling between the reductant (donor, D) and oxidant (acceptor, A) $\left(T_{\mathrm{DA}}\right.$, also written as $H_{\mathrm{DA}}$ or $\left.H_{\mathrm{AB}}\right)$

$$
k_{\mathrm{ET}}=\frac{4 \pi^{2}}{h \sqrt{4 \pi \lambda R T}} T_{\mathrm{DA}}^{2} e^{-\frac{\left(\Delta G^{0}+\lambda\right)^{2}}{4 \lambda R T}}
$$

where the remaining symbols have the usual meaning. The simplest representation for $T_{\mathrm{DA}}$ states an exponential decay with distance $r$ between D and A (Hopfield, 1974)

$$
k_{\mathrm{ET}}=k_{0} e^{-\left[\beta\left(r-r_{0}\right)\right]} e^{-\frac{\left(\Delta G^{0}+\lambda\right)^{2}}{4 \lambda R T}}
$$

where $r_{0}$ is the van der Waals contact distance and $k_{0}$ is the maximum ET rate when D and A are in van der Waals contact $\left(r=r_{0}\right)$ and $\lambda=-\Delta G^{\circ}$. Parameter $\beta$ in equation 2 is the decay factor, which measures the nature of the intervening medium with respect to its efficiency to mediate ET (Page et al., 1999).

When studying inter-protein and/or intra-protein ET processes in oxidoreductases, it is important to determine all the essential parameters involved in equations 1 and 2. This information, together with kinetic, biochemical, and structural data, is necessary to understand in detail how the enzyme works. Experimentally, the value of $T_{\mathrm{DA}}$ could be measured by UVvis/NIR spectroscopies using the known Hush formalism for the case where the ET system presents a metal to metal charge transfer reaction, as it occurs in mixed-valence complexes (Creutz, 1983). This is not possible for the systems studied here, since the charge transfer band discussed above for Nir is between the $\mathrm{Cu}$ (II) ion and the S-Cys of T1, whereas Aor does not show any charge transfer band. When the enzyme has a redox center that can be independently spectroscopically monitored (UV-vis absorption, Raman, or EPR spectroscopies), the technique of choice to study ET processes is Laser Flash Photolysis (LFP) (formerly Pulse Radiolysis), provided that the process is amenable to light triggering. Performing LFP experiments at different temperatures allows one to least-squares fit equation 1 to the experimental data in order to determine $\Delta G^{\circ}, \lambda$ and $T_{\mathrm{DA}}$ (Davidson, 1996; Davidson, 2002; Davidson, 2008; Formosinho and Barroso, 2012). Next, equation 2 is fit to the same dataset, but fixing the previously determined parameters $\Delta G^{\circ}$ and $\lambda$. Through this procedure, parameters $\beta$ and $r$ can be determined. Given the large number of parameters to be determined, this approach applied to complex systems like oxidoreductases may conduct to erroneous interpretations of the intra-protein electron transfer process at molecular level (Davidson, 2002; Formosinho and Barroso, 2012). This fact determines that the characterization of the intraprotein ET pathway in these enzymes must be studied also through other experimental techniques. In the next sections we will discuss other techniques to determine parameters $\Delta G^{\mathrm{o}}$ and $T_{\mathrm{DA}}$ independently, or at least to estimate their values using electrochemical and spectroscopic tools, and how ET processes in an oxidoreductase can be predicted and studied.

\section{Determination of the driving force $\left(\Delta G^{\circ}\right)$ by electrochemical methods}

Understanding the oxidoreductase catalytic mechanism and its relationship with the intramolecular ET process requires establishing the reduction potentials of the metal centers situated along the ET pathway, e.g. those for T1 and T2 for NirK, and $\mathrm{Mo}, \mathrm{FeS} 1$, and FeS2 for Aor. As shown in equations 1 and 2, the ET rate $\left(k_{\mathrm{ET}}\right)$ depends on $\Delta G^{\mathrm{o}}$, which is related to the reduction potentials of the intervening redox centers of the oxidoreductase by

$$
\Delta G^{0}=-n F \Delta E^{0}
$$


where $n$ is the number of electrons involved in the reaction and $F$ is the Faraday constant. $\Delta E^{\mathrm{o}}$, also known as $E_{\text {cell }}$, is equal to the reduction potential $\left(E^{\circ}\right)$ of oxidant $A$ minus the reduction potential of reductant $D . E^{\mathrm{o}}$ is the half-cell potential or half-reaction reduction potential under standard conditions, i.e. $298 \mathrm{~K}, 1 \mathrm{~atm}, 1 \mathrm{M}$ solutions. For most proteins, it is impossible to work at such concentrations and, therefore, midpoint reduction potentials $\left(E_{\mathrm{m}}\right.$ or $E^{\mathrm{o}}$ ') of the redox cofactors are determined. $E_{\mathrm{m}}$ values can be experimentally determined through different electroanalytical methods, such as redox potentiometry and voltamperometry.

$E_{\mathrm{m}}$ values of several redox cofactors in metalloproteins have been determined using voltamperometric techniques like cyclic voltammetry, differential pulsed voltammetry (aka polarography) and square-wave voltammetry. For instance, we have determined the $E_{\mathrm{m}}$ of two physiological redox partners of NirK (Sinorhizobium meliloti Paz and Bradyrhizobium japonicum CytC) by cyclic voltammetry (Ferroni et al., 2014). The experimental setup for this technique consists of three electrodes (working, reference, and auxiliary) immersed in an electrolyte buffered solution (Figure 2A). The redox protein adsorbed on the working electrode must be capable of exchanging electrons with the solid conducting material used. The applied potential is swept across a given range, and the current is measured. For a thorough explanation of direct electrochemistry of metalloproteins, see Leger et al. (Leger and Bertrand, 2008).

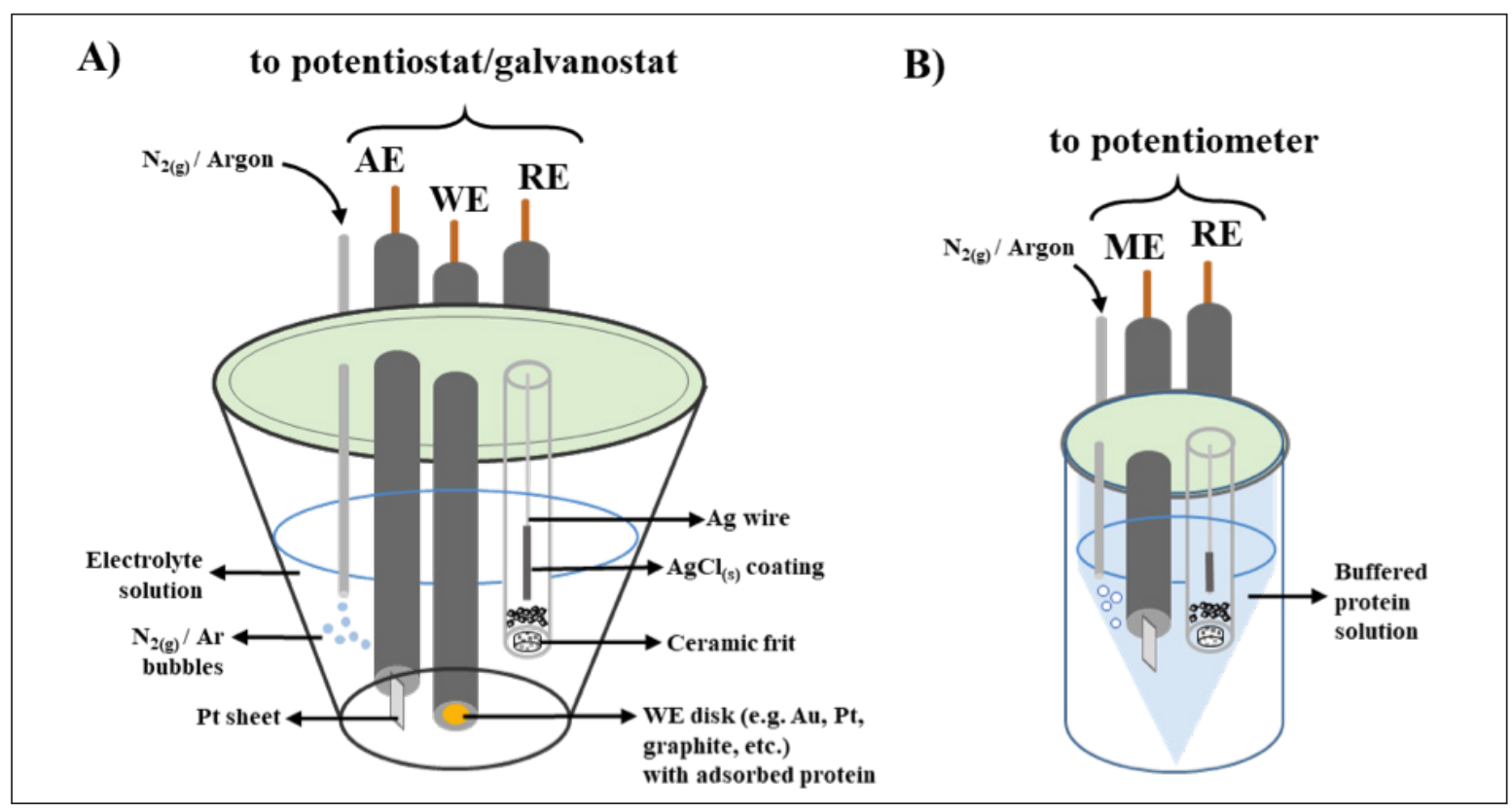

Figure 2. Three- and two-electrode cell setups used for voltamperometric (A) and potentiometric (B) measurements. WE, RE, AE and ME stand for Working, Reference, Auxiliary and Measuring Electrodes, respectively.

Voltamperometric techniques may present some technical problems, such as an inefficient ET between the metalloprotein and the working electrode, which may be overcome by spectropotentiometry. In contrast to voltamperometry, spectropotentiometry is an equilibrium technique in which the oxidoreductase in buffered solution is poised at several potentials by adding reducing (e.g. sodium dithionite or ascorbate) or oxidizing (e.g. potassium ferricyanide or hexachloroiridate) reagents. Solution potential at equilibrium is measured by a setup of two electrodes, comprising a measuring electrode (which must be a noble material like $\mathrm{Au}$ or $\mathrm{Pt}$ ) and a reference electrode $(\mathrm{Ag} / \mathrm{s}) / \mathrm{AgCl}$ or $\mathrm{Hg}_{(1)} / \mathrm{Hg}_{2} \mathrm{Cl}_{2}$ ) (Figure 2B)

To understand how spectropotentiometry works, we will consider the simplest redox equilibrium

$$
\mathrm{Ox}+\mathrm{e}^{-} \rightarrow \mathrm{Red}
$$

where Ox and Red stand for the oxidized and reduced species, respectively. Let us assume that Ox and/or Red can be quantified by any spectroscopic technique and that the electron involved in the half reaction is provided by a redox partner that is spectroscopically silent or does not interfere in the determination of the Ox/Red fraction. 
Considering the Nernst equation for the redox half reaction and that the total concentration of the redox cofactor is $[$ Total $]=[\mathrm{Ox}]+[\mathrm{Red}]$, obtaining the change in Ox and Red concentration with solution potential $(E)$ is straightforward:

$$
[\mathrm{Ox}]=\frac{[\text { Total }]}{1+e^{\frac{n F}{R T}\left(E-E^{0}\right)}}
$$

To determine the signal intensity of Ox vs. E, a specific spectroscopic feature of the redox center must be monitored by techniques such UV-Vis absorption, EPR, CD, MCD, Fluorescence, NMR, FT-IR and RAMAN. The spectroscopic properties of the redox center (e.g. chromophore, paramagnet, etc.) determine the technique and whether the oxidized or reduced form is measured. We will explain below the UV-vis and EPR monitored potentiometry results we obtained with the two oxidoreductases described in this review.

\subsection{UV-vis absorption spectroscopy}

UV-vis absorption spectroscopy is one of the most useful and easy to apply (as regards costs and operator training) spectroscopic techniques to monitor redox cofactors. The optical spectra of metalloproteins give information on the electronic structure of redox cofactors, and the technique can also be used to study the absorbance of a specific chromophore as a function of a physicochemical variable such as concentration, $\mathrm{pH}, E$, etc.

For potentiometric titrations monitored by UV-vis spectroscopy, the intensity of an absorption band or peak is related to the species concentration by the Beer-Lambert law. The common setup for determining the absorbance at different $E$ values involves, as depicted in Figure 3A, an inert atmosphere vessel allowing the simultaneous measurement of $E$ and the absorbance of the protein solution. A single wavelength or the entire absorption spectrum can be recorded at each stable $E$ value. The intensity of a characteristic absorption peak of the redox center of interest can be plotted against $E$ and equation 4 can be fit to the experimental dataset.
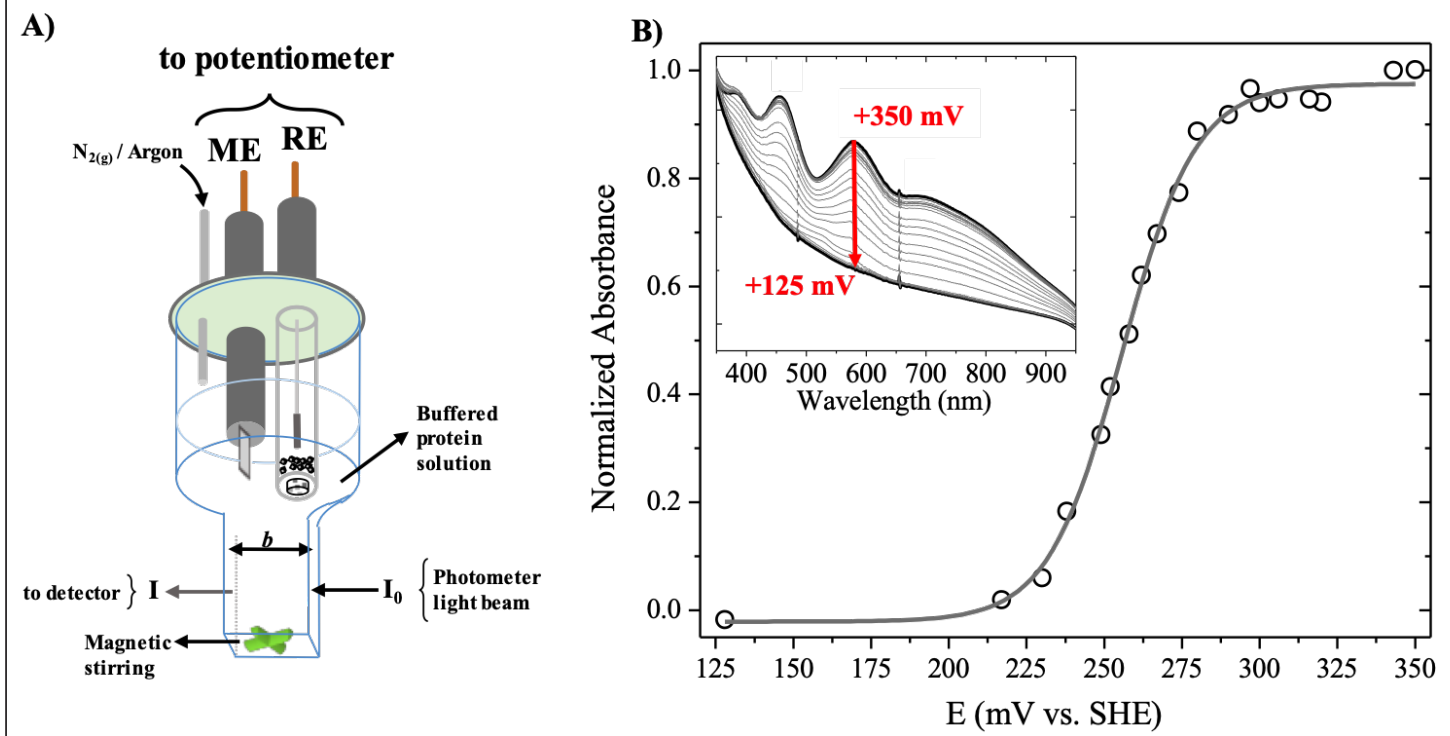

Figure 3. A) modified UV-vis quartz cuvette used for potentiometric titrations. B) Absorbance of NirK oxidized-T1 center at $590 \mathrm{~nm}$ as a function of the solution potential. Equation 4 was fit to the data to yield $E_{\mathrm{m}}=+250 \mathrm{mV}$. The inset shows the UV-vis absorption spectra of oxidized T1 of Nir for different potentials. 
Panel B in Figure 3 shows a potentiometric titration of NirK monitored by UV-vis spectroscopy. Both the titration and absorbance measurements were simultaneously performed at $298 \mathrm{~K}$ and pH 6.0, yielding $E_{\mathrm{m}}=+250 \mathrm{mV}$ (vs. SHE). Even though this procedure is useful to determine the T1 reduction potential of NirK, the ET driving force $\left(\Delta G^{\circ}\right)$ can only be calculated knowing both $\mathrm{T} 1$ and $\mathrm{T} 2$ reduction potentials, which cannot be evaluated by this procedure, as T2 does not present UV-vis absorption bands. The next EPR section will show how to measure both.

\subsection{EPR spectroscopy}

UV-vis spectroscopy is by far the simplest technique to perform a spectropotentiometric titration, but it cannot be used in cases of very weak or undetectable absorption bands, as is the case of the T2 copper site in NirK and the Mocofactor in Aor. Another example of the limitation of this technique is the case of the FeS centers in Aor, which show overlapped broad absorption bands that cannot be discriminated. In these cases, EPR was shown to be the technique of choice. The advantage of EPR is that most important redox active ions of life (Fe, $\mathrm{Cu}$, Mo and W) can generally be well discriminated through their typical EPR signatures and properties, allowing the determination of $E_{\mathrm{m}}$ values by poising the protein solution at several potentials with reducing and/or oxidizing reagents. Furthermore, EPR can provide important information in those systems containing at least two paramagnetic cofactors presenting intercenter magnetic interactions. The EPR signals in the examples below will be used, as in the case of the UV-vis spectra, as a simple fingerprint of the cofactors. Readers interested in understanding more deeply the information that can be obtained from an EPR experiment can read (Rizzi et al., 2016) and references therein.

The T2 center of NirK is paramagnetic in the cupric state and, therefore, can be monitored by EPR spectroscopy. Figure 4 (Top) shows two EPR spectra of NirK. In the as-prepared form, both T1 and T2 are in their oxidized $\mathrm{Cu}^{2+}$ state ( $\mathrm{d}^{9}$, spin $S=1 / 2$ ), giving rise to nearly axial overlapped EPR signals (Spectrum A), which are distinguishable through their hyperfine coupling constant $A_{\|}\left(A_{\|}^{\mathrm{T} 1}<A_{\|}^{\mathrm{T} 2}\right)$. This feature allows simulating each spectrum separately, and also to quantify $\mathrm{T} 1$ and T2 concentrations; EPR parameters can be found in (Cristaldi et al. 2018). Upon incubation with sodium ascorbate $(E \sim 0 \mathrm{mV})$, T1 becomes EPR silent, as it is reduced to $\mathrm{Cu}^{1+}\left(\mathrm{d}^{10}, S=0\right)$, whereas a fraction of T2 remains in the $\mathrm{Cu}^{2+}$ oxidation state (Figure 4, spectrum B). In contrast, no EPR signals are detected upon addition of a 10-fold molar excess of sodium dithionite under anaerobic conditions $(E \sim-660 \mathrm{mV}$, not shown), indicating that both copper centers are reduced to the cuprous oxidation state. Ferricyanide addition to as-prepared NirK $(E \sim+440 \mathrm{mV})$ does not modify the intensity and line shape of the EPR signal, indicating that both T1 and T2 are fully oxidized in the as-prepared sample (not shown). This relatively simple experiment allows inferring that the T1 reduction potential is higher than that of T2, and that the reduction potentials of both centers fall within the range +440 to $0 \mathrm{mV}$ (Olesen et al., 1998; Pinho et al., 2004). Thus, this approach is useful in those cases where it is not possible to perform an EPR-monitored redox titration, for example because of limitations in sample amount.

In contrast to UV-vis absorption spectroscopy, metal centers EPR signals are recorded at cryogenic temperatures. Therefore, EPR spectroscopy does not allow simultaneously measuring solution potential and recording a spectrum. For this reason, in order to perform an EPR-monitored redox titration, samples of the protein solution poised at different $E$ values at room temperature and under an $\mathrm{O}_{2}$-free atmosphere, must be withdrawn, frozen to liquid nitrogen temperature, and measured afterwards. Note that, even though EPR measurements are performed at cryogenic temperatures due to experimental reasons, spectra correspond to samples poised at room temperature potential. Figure 4 (bottom) shows a redox potentiometric titration of NirK monitored by CW-EPR, together with the EPR spectra at different potentials in the inset. In this case, the intensity of the EPR signal observed comprises both T1 and T2 signals. A plot of signal intensity (area below integrated spectra) vs. solution potential $(E)$ yielded a double sigmoid curve that was fit to the sum of two one-electron Nernst equations. The $E_{\mathrm{m}}$ values obtained at $\mathrm{pH} 7.0$ were +224 and $+108 \mathrm{mV}$ (vs. SHE) for T1 and T2, respectively.

Before going to the EPR analysis of Aor, just a brief explanation regarding the electronic structure of Mo and FeS metal centers. Molybdenum in Aor can be found in three different oxidation states, $\mathrm{Mo}^{6+}, \mathrm{Mo}^{5+}$, and $\mathrm{Mo}^{4+}$. Only $\mathrm{Mo}^{5+}\left(\mathrm{d}^{1}, S=\right.$ $1 / 2$ ) is detectable by EPR and gives rise to signals with all $g$-values lower than 2 . The two [2Fe-2S] centers can be found in two oxidation states. The oxidized state $\left([2 \mathrm{Fe}-2 \mathrm{~S}]^{2+}\right)$ is EPR silent as it contains two strongly antiferromagnetically coupled $\mathrm{Fe}^{3+}$ ions with a ground state with $S=0$, which is the only one thermally populated under the EPR experimental conditions. The $[2 \mathrm{Fe}-2 \mathrm{~S}]$ center becomes paramagnetic on reduction of one of the $\mathrm{Fe}^{3+}$ ions to $\mathrm{Fe}^{2+}$. The resulting $\mathrm{Fe}^{2+}-\mathrm{Fe}^{3+}$ pair $\left([2 \mathrm{Fe}-2 \mathrm{~S}]^{1+}\right)$ is also strongly antiferromagnetically coupled, but with a $S=1 / 2$ ground state and hence detectable by EPR (Johnson, 1998). 

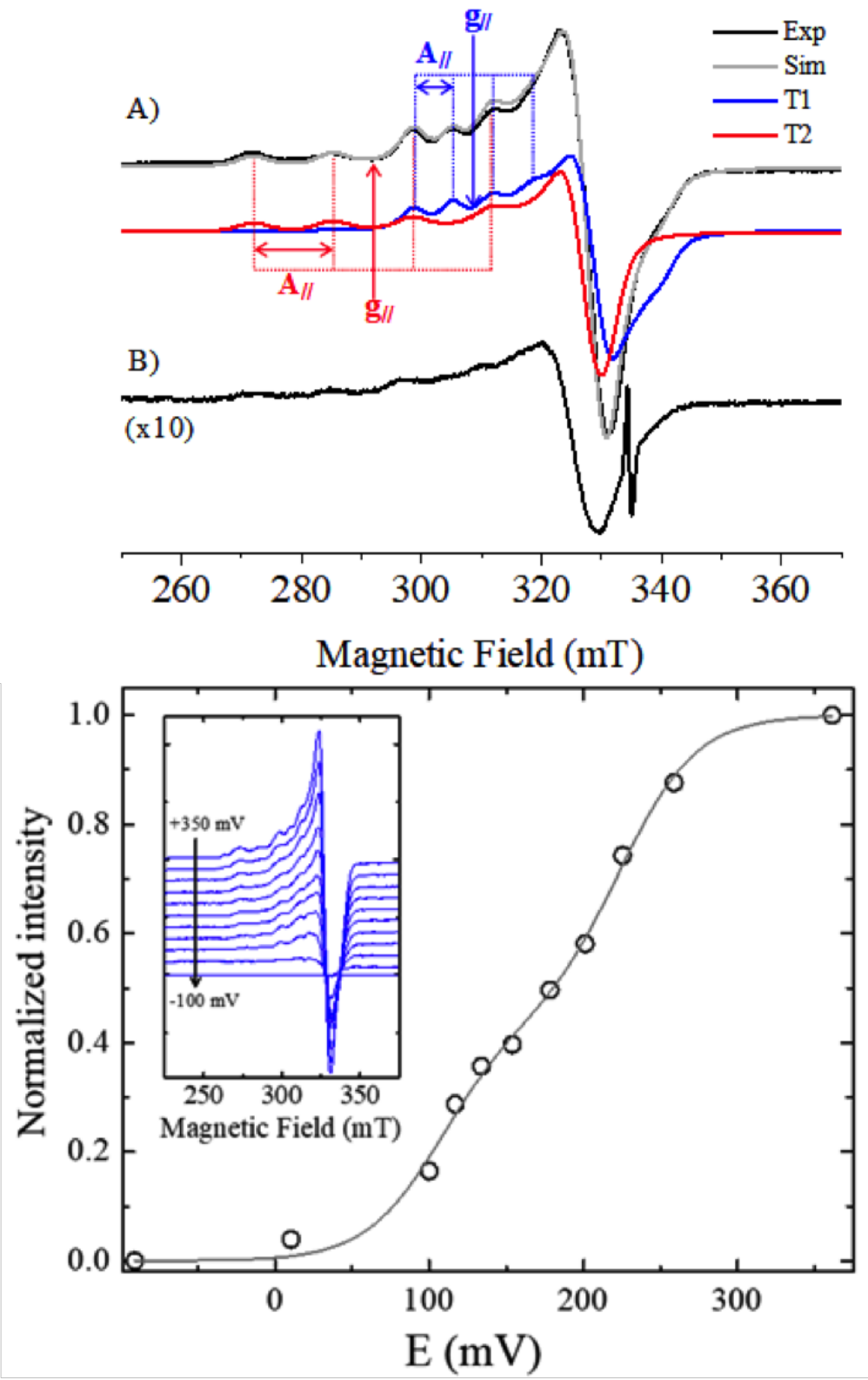

Figure 4. EPR signals (top) in the as-prepared (spectrum A) and ascorbate-reduced form (spectrum B) of NirK. Experimental spectra are in black; simulation of spectrum A is in gray and it was obtained by adding T1 and T2 simulations (blue and red, respectively). The EPR parameters indicated on the figure can be found in (Cristaldi et al., 2018). Redox potentiometric titration of NirK from $S$. meliloti monitored by EPR (bottom). The solid line was obtained by least squares fitting the sum of two-independent Nernst equations with $\mathrm{n}=1$ to the data. EPR spectra as a function of $E$ are shown in the inset. The midpoint reduction potentials obtained were +224 and $+108 \mathrm{mV}$ for $\mathrm{T} 1$ and $\mathrm{T} 2$, respectively (Ferroni et al. 2014).

In contrast to NirK, redox cofactors of Aor are EPR-silent in the as-prepared form, and EPR signals are only developed upon dithionite addition under strict anaerobic conditions. Dithionite reduction of Aor for 20 min under anaerobic conditions ( $E \sim-660 \mathrm{mV}$ vs SHE, reduction for a time lower than 20 min gives rise to a different EPR signal) gives rise to EPR signals associated with the $\mathrm{Mo}^{5+}$ ion and the two $[2 \mathrm{Fe}-2 \mathrm{~S}]^{1+}$ clusters (Figure 5). Using the same arguments given above in this section for estimating reduction potentials in $\mathrm{Cu}(\mathrm{II})$ centers of NirK, we can conclude that metal centers reduction potentials in Aor are below $0 \mathrm{mV}$ (vs. SHE). 


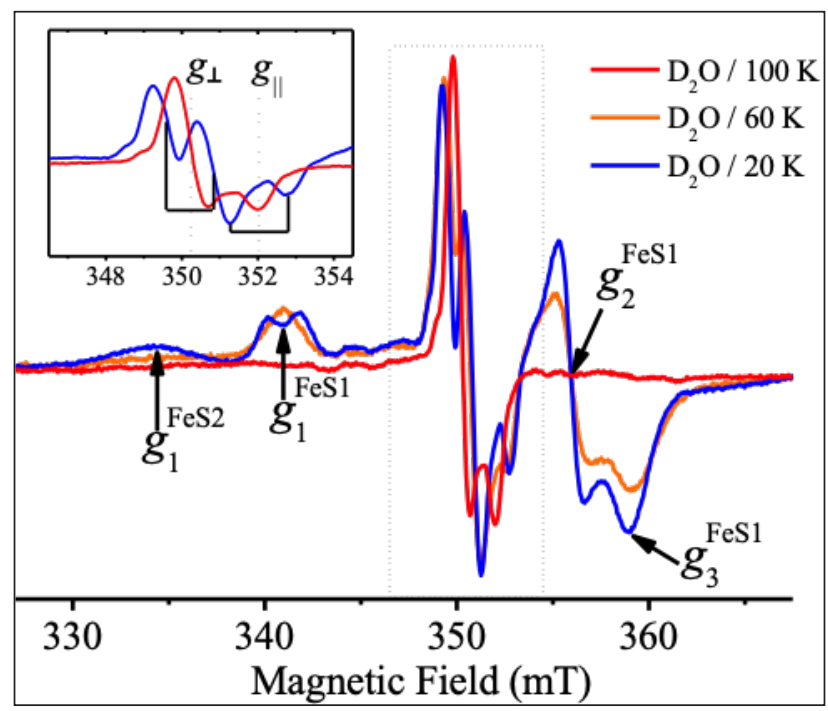

Figure 5. EPR signals of $\mathrm{D}_{2} \mathrm{O}$-exchanged Aor upon dithionite addition at different temperatures. The inset shows the $\mathrm{Mo}^{5+}$ signals at $100 \mathrm{~K}$ and $20 \mathrm{~K}$. Note the nearly isotropic splitting of $\mathrm{Mo}^{5+} \mathrm{EPR}$ features at low temperature originated by Mo-proximal FeS center magnetic coupling. EPR parameters indicated on the figure with arrows can be found in (González et al., 2009) and references therein.

The $\mathrm{Mo}^{5+}$ signal obtained under these conditions is commonly named "slow-type" in the literature on Mo-enzymes (red and blue spectra in the inset on Figure 5) (Hille, 1996), but hereafter it will be referred to as $\mathrm{Mo}^{5+}$ EPR signal. Whereas $\sim 100 \%$ of the FeS centers are paramagnetic at this solution potential, only about $10-15 \%$ of the total molybdenum is obtained as $\mathrm{Mo}^{5+}$ species. This indicates that at this potential, FeS centers were fully reduced to the paramagnetic [2Fe$2 \mathrm{~S}]^{1+}$ state, indicating that the $E_{\mathrm{m}}$ values of both FeS centers fall in the range of $-450-0 \mathrm{mV}$ (vs SHE). A similar analysis, but with some added complexity, can be performed for Mo. The fact that only $10-15 \%$ of $\mathrm{Mo}^{+5}$ species are detected by EPR indicates that the $E_{\mathrm{m}}$ values of the $+6 /+5$ and $+5 /+4$ couples fall in the same range, and also that both $E_{\mathrm{m}}$ values are very close.

After analyzing the EPR spectra of the system, i.e., after the distinct EPR signals are identified, the redox titration can be performed. The $E_{\mathrm{m}}$ values of FeS centers can be obtained as explained for NirK. To analyze the $E_{\mathrm{m}}$ values associated with the redox transitions of the Mo cation, the redox equilibriums of both couples should be taken into account, as the redox behavior of Mo species depends on the couples $\mathrm{Mo}^{6+} / \mathrm{Mo}^{5+}$ and $\mathrm{Mo}^{5+} / \mathrm{Mo}^{4+}$. This analysis yielded $E_{\mathrm{m}}$ values of -450 and $-530 \mathrm{mV}$ for the $\mathrm{Mo}^{6+} /{ }^{5+}$ and $\mathrm{Mo}^{5+} /{ }^{4+}$ couples, respectively, for $D$. gigas Aor, and, in addition, it was possible to determine $E_{\mathrm{m}}=-280$ and $-285 \mathrm{mV}$ for FeS1 and FeS2, respectively (Moura et al., 1978).

It should be noted that the $\Delta E^{0}$ values for the pair T1/T2 obtained for NirK are uphill, i.e. in contrast of the electron flow experienced during catalysis. It is not uncommon to obtain such results in metalloproteins, and some explanations have been assayed stating that an uphill step can be compensated for diminishing the DA distance (Moser et al., 2010). In the case of the NirK enzyme studied by us, there is strong evidence that the $E_{\mathrm{m}}$ of the $\mathrm{Cu}$ centers are modulated by the interaction of the enzyme with the substrate and the physiological ED during catalysis, giving rise to a downhill ET process $\left(\Delta E^{0}>0 \mathrm{mV}\right)$ (Ferroni et al., 2014).

\section{Assignment of the spectroscopically detected metal centers to those observed in the protein structure}

The assignment of the spectroscopically detected metal centers to those observed in the X-ray structure is an essential step in characterizing proteins that contain pathways composed of various metal cofactors. This problem is simple in proteins with metal centers showing distinguishable spectroscopic features, e.g. the two Cu centers in NirK, where only T1 shows UV-Vis absorption bands (Figure 3B, inset), and T1 and T2 can be easily discriminated by EPR (Figure 4 top). 
However, the solution is not trivial for proteins such as Aor, which has two very similar [2Fe-2S] centers. This problem can be solved using different strategies. The most common approach is to generate site-directed variants by molecular biology techniques, changing an amino acid residue that acts as a ligand of the redox cofactor, and then verifying which spectroscopic component was modified. In the case of Aor, the generation of site-directed variants was not possible. Therefore, assignment was based on the analysis of the weak magnetic interaction between Mo and the proximal FeS cluster that splits the $\mathrm{Mo}^{5+}$ EPR signal at low temperatures (Figure 5). This involves detecting which FeS (1 or 2) EPR signal is associated with the splitting of the $\mathrm{Mo}^{5+}$ spectrum. This can be achieved using EPR by poising an Aor sample at a potential value between the reduction potentials of the FeS centers in order to obtain different populations of reduced (paramagnetic) and oxidized (diamagnetic) FeS centers. This experiment was performed in D. alaskensis Aor, which presents more separated $E_{\mathrm{m}}$ values, which is not the case of D. gigas Aor. Once that the FeS EPR signal responsible for the $\mathrm{Mo}^{5+}$ EPR splitting is identified, the correlation is straightforward, as the $\mathrm{Mo}^{5+}$ EPR splitting must be provoked, as said above, by the crystallographically identified proximal FeS center (Figure 1). Thus, it was possible to conclude that the EPR signals identified as FeS1 and FeS2 correspond to the proximal and distal FeS centers of Aor, respectively. A thorough explanation about how this method was performed can be found in references (Andrade et al., 2000; Rizzi et al., 2016). Similar conclusions for Aor, but using an alternative strategy also based on the Mo ${ }^{5+}$ EPR signal splitting provoked by the proximal FeS center, consisted of simulating $\mathrm{Mo}^{5+} \mathrm{EPR}$ signal splitting as a function of temperature. The dependence of $\mathrm{Mo}^{5+}$ splitting on temperature (see Figure 5) is due to the higher relaxation rate of FeS1 in the pair FeS1$\mathrm{Mo}^{5+}$, a characteristic that is present in all magnetically interacting pairs of spins presenting different relaxation rates. Ideas behind this issue, full experimental data for Aor, as well as the mathematical formalism employed in their analysis can be found in (González et al., 2009) and references therein.

\section{Evaluating the integrity of the ET pathway upon inhibition}

Enzyme inhibition studies are mandatory to understand enzyme catalytic mechanisms. For oxidoreductases, as for any other class of enzymes, inhibition can be either irreversible or reversible. The latter can be competitive, uncompetitive, or non-competitive. The type of inhibition can be unambiguously determined through in-depth steady state enzyme kinetic studies (Cornish-Bowden 2004). Nevertheless, spectroscopic studies are needed to understand at a molecular level how enzyme inhibition occurs, and to gain insight into catalytic mechanisms. There is no specific methodology to study whether inhibition is due to disruption of the ET pathway. Hence, we will explain specific strategies we followed in our examples to evaluate integrity of the ET pathway upon inhibition.

Aor presents the peculiarity that the active site yields redox inert $\mathrm{Mo}^{5+}$ species upon adding enzyme inhibitors such as arsenite, ethylene glycol, and glycerol (Santos-Silva et al. 2009). This fact determines that Aor can be obtained in two states, in which Mo is always as $\mathrm{Mo}^{5+}$ but the FeS clusters can be either in their diamagnetic (oxidized) or paramagnetic (reduced) states. In other words, the $\mathrm{Mo}^{5+}$ species can be measured by EPR in these two enzyme states, one in which the $\mathrm{Mo}^{5+}-\mathrm{FeS} 1$ coupling is "switched off" (oxidized form, diamagnetic FeS1), and a second where the coupling is "switched on" (reduced form, paramagnetic FeS1) (Boer et al., 2004; Santos-Silva et al., 2009). The experiment consists in measuring the intensity of the $\mathrm{Mo}^{5+}$ signal as a function of microwave power to evaluate the degree of saturation of the EPR signal. The higher the microwave power, the higher the probability that the EPR signal attains saturation. Another concept is necessary to understand the saturation EPR experiment. Magnetic coupling of the $\mathrm{Mo}^{5+}$ species with a nearby faster relaxing paramagnetic species enhances the relaxation rate of the slow-relaxing species. With these concepts in mind, it is easy to understand the saturation EPR experiment. The reduced sample of inhibited Aor (magnetic interaction "on") requires higher power to reach saturation due to the enhanced relaxation rate of the $\mathrm{Mo}^{5+}$ ion induced by the faster relaxing FeS1 center. This fact indicates that the Mo-FeS1 ET pathway can transmit magnetic interaction in the enzyme inhibited form, confirming that the ET pathway is not affected upon inhibition. Details on how to perform this experiment can be found in (Boer et al., 2004; Rizzi et al., 2016).

For NirK, the saturation strategy could not be used due to two simple reasons. First, no T1-T2 magnetic coupling is observable. Second, the two copper centers show similar relaxation rates. Therefore, to evaluate the integrity of the ET pathway in NirK, we exploited the $\mathrm{pH}$ dependence of its catalytic activity (Cristaldi et al., 2018). NirK presents maximal activity at $\mathrm{pH} 5-6$ and no activity at $\mathrm{pH}>8$, in which the dependence with $\mathrm{pH}$ could be attributed the $\mathrm{N}^{\delta 1} \mathrm{H}$... $\mathrm{O}=\mathrm{C}$ hydrogen bond present in the T1-T2 ET pathway (Figure 1). To prove this hypothesis, we performed kinetic studies of $S$. meliloti NirK at $\mathrm{pH}$ of maximal and minimal activity using dithionite as electron donor in which the starting and final oxidation states of the copper centers were monitored by UV-Vis and EPR spectroscopies. Both spectroscopies showed that at $\mathrm{pH}$ 6 both $\mathrm{T} 1$ and $\mathrm{T} 2$ center are as $\mathrm{Cu}^{2+}$ upon redox cycling the enzyme upon nitrite addition. In contrast, at pH 10 only T2 is reoxidized to $\mathrm{Cu}^{2+}$, which confirms that the lack of activity at high $\mathrm{pH}$ may be due to an innefficient/non-existent ET. The conclusion that emerges from this experiment is that the Cys-His covalent pathway (marked in blue in Figure 1) is not 
involved in ET since it is $\mathrm{pH}$-independent, suggesting that the ET occurs through the pH-dependent green path in Figure 1. Another methodology used to evaluate ET pathway integrity will be discussed below under section 8 .

\section{Relation between electronic coupling $\left(T_{\mathrm{DA}}\right)$ and the isotropic exchange constant $J$}

An application of the Marcus theory is to predict the $k_{\mathrm{ET}}$ between two distant redox centers bridged by a chemical pathway (Marcus and Sutin 1985). If the two redox centers are paramagnetic and coupled by isotropic exchange $(J)$, the bridging chemical pathway not only mediates ET, but also transmits isotropic exchange (Calvo et al., 2000) (Note: isotropic exchange, also known as superexchange, is the main Mo-FeS1 interaction that contributes to the splitting of the $\mathrm{Mo}^{+5} \mathrm{EPR}$ signal, Figure 5; despite it is not magnetic in origin, it is usually regarded as a magnetic interaction). For large distances between spins, the term $T_{\mathrm{DA}}$, which depends on the nature of the chemical pathway bridging two redox centers, is related to the isotropic exchange coupling constant by

$$
J \approx-T_{\mathrm{DA}}^{2 / \mathrm{U}}
$$

where $\mathrm{U}$ is the difference in energy between the chemical species $\mathrm{DA}$ and $\mathrm{D}^{+} \mathrm{A}$; with the latter being obtained when one electron is removed from $\mathrm{D}$ and transferred to A. Note also that this expression is valid for $T_{\mathrm{DA}}<<\mathrm{U}$. Thus, we can see how the evaluation of $J$ by EPR is related to the term $T_{\mathrm{DA}}$ that appears in equation 1.

Aor is a representative example in which the evaluation of $J$ by EPR can be useful to study the factors that govern ET between redox centers in metalloproteins. The catalytic mechanism of Aor involves substrate binding followed by a twoelectron oxidation of the $\mathrm{Mo}^{4+}$ center once the product is released (Brondino et al., 2006). The structure of as-prepared Aor corresponds to the enzyme in the ready-state and is the starting point of the catalytic cycle (Figure 1, bottom), whereas that of alcohol-inhibited Aor (with the alcohol moiety being bound to the $\mathrm{Mo}^{5+}$ ion) resembles a situation where the product of the reaction (the corresponding acid, Figure 1) is formed and remains within bonding distance with the Mo ion (SantosSilva et al., 2009).

As for the $\mathrm{Mo}^{5+} \mathrm{EPR}$ signal obtained from the enzyme resting state (Figure 5 and spectrum A in Figure 6), the alcoholinhibited Aor $\mathrm{Mo}^{5+}$ EPR signal is also split by magnetic interaction with the proximal FeS center (Figure 6). The splitting of the EPR lines of glycerol- and ethylene glycol-reacted Aor increases notably relative to that of reduced as-prepared Aor.

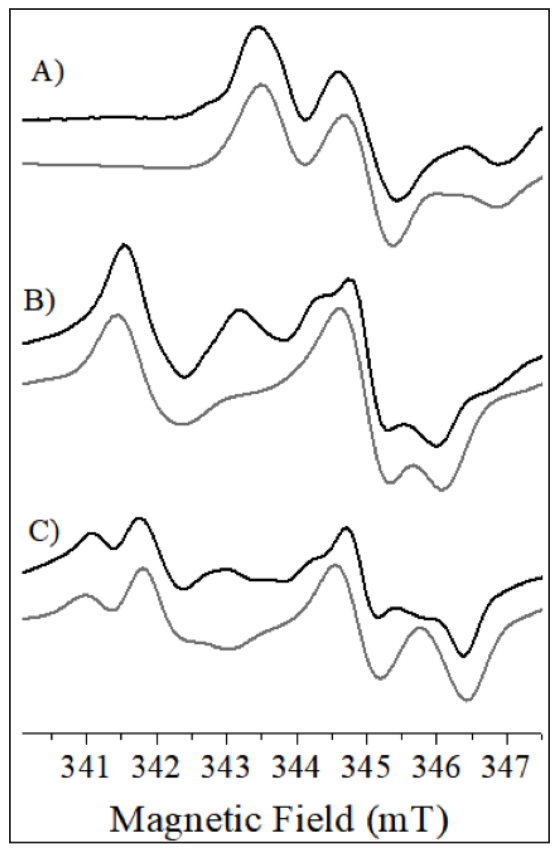

Figure 6. EPR spectra (black) at $20 \mathrm{~K}$ for the $\mathrm{Mo}^{5+}$ center of reduced as-prepared Aor (spectrum A), and ethylene glycoland glycerol-reacted Aor (spectra B and C, respectively) with simulations (gray). Simulations yielded $J=-16.6 \times 10^{-4}$ $\mathrm{cm}^{-1}$ for as-prepared Aor and $J=-33.3 \times 10^{-4} \mathrm{~cm}^{-1}$ for ethylene glycol- and glycerol-reacted Aor. Simulation details can be found in reference (Gómez et al., 2015). 
The $\approx 2$-fold increase in $J(J$-values are in the caption to Figure 6$)$ upon alcohol binding indicates that the capability to transmit isotropic exchange is enhanced, likely due to structural changes in the Mo-FeS1 pathway, or changes in the electronic structure of either $\mathrm{Mo}^{5+}$ or FeS1. High resolution structural data of alcohol-inhibited Aor showed no changes associated with the pyranopterin chemical pathway, and the FeS1 EPR signal is identical to that of as-prepared Aor reduced with dithionite (Santos-Silva et al., 2009). Therefore, the increase in $J$-value would indicate changes in the electronic structure of $\mathrm{Mo}^{5+}$, which is a reasonable hypothesis considering the change of $\mathrm{Mo}^{5+}$ geometry produced after alcohol binding. This hypothesis was addressed by computational calculations that showed that coordination of the alcohols led to a redistribution of the electronic spin density at $\mathrm{Mo}^{5+}$ and its ligands (Gómez et al., 2015). It is important to note that although these studies are not performed during catalysis, they allow one to obtain clues about how ET processes are carried out in these enzymes. For the resting state, calculations showed that part of the spin density is displaced towards the catalytically relevant $\mathrm{OH}_{x}$ ligand, whereas the spin density is displaced towards the dithiolene sulfur ligands of the pterin moiety in the situation that emulates product formation. This fact suggests that this spin density reorganization would raise $T_{\mathrm{DA}}$ upon product formation during catalysis, and thus favoring the ET process towards the FeS centers, a process which is required to return the enzyme to the original resting state.

\section{Site-directed mutagenesis as a tool in the study of ET pathways}

Specific structure-function relationships can be elucidated by site-directed mutagenesis, as this molecular biology tool can be useful to understand how certain amino acid residues of the polypeptide chains modulate ET rates. As an example about how a chemical pathway can be altered by site-directed mutagenesis, the Cys-His ET pathway of $S$. meliloti NirK was modified. As shown in Figure 1, this pathway has two subpaths, one that involves a pure covalent chemical pathway (highlighted in blue) constituted by the protein backbone and the Cys and His side chains, and another one that is a mixed chemical pathway constituted by a His- $\mathrm{N}^{\mathrm{d} 1} \mathrm{H} . . \mathrm{O}=\mathrm{C}-\mathrm{Cys}$ hydrogen bond that partially shortcuts the T1-T2 bridging covalent link (highlighted in green, Figure 1). We constructed two variants of S. meliloti NirK, H171D and C172D (Note: H171D and C172D are two protein variants where the residues histidine 171 and cysteine 172 were changed to aspartic acid), and their kinetic, structural, and spectroscopic properties were compared with those of the wild type enzyme (Cristaldi et al., 2018). The H171D variant (Figure 7) contains both T1 and T2 only connected by the pure covalent subpath and, even though it retains its ability to bind the substrate, it is unable to catalyze nitrite reduction. In contrast, the $\mathrm{C} 172 \mathrm{D}$ variant contains two copper sites, one of them being similar to $\mathrm{T} 2 \mathrm{Cu}$ in the wild type enzyme. The cysteine variant can reduce nitrite using artificial electron donors, which confirms that, though with less efficiency, the reaction can be conducted entirely at the active site without mediation of the Cys-His ET pathway. From kinetic studies on the wild type enzyme and C172D, it is evident that the involvement of the Cys-His chemical pathway increases enzyme efficiency for catalysis. This is not the case of H171D, where the modification of the ET pathway, more specifically the lack of the His- $\mathrm{N}^{\delta 1} \mathrm{H} \ldots \mathrm{O}=\mathrm{C}$-Asp hydrogen bond bridge, was suggested to be the cause for the lack of catalytic activity. In summary, site-directed mutagenesis is useful for modifying ET pathways selectively and hence demonstrate the relevance of certain chemical bonds in intra-protein ET. Readers interested in reading about alternative applications of this technique related to ET studies can see reference (Davidson, 2008). 


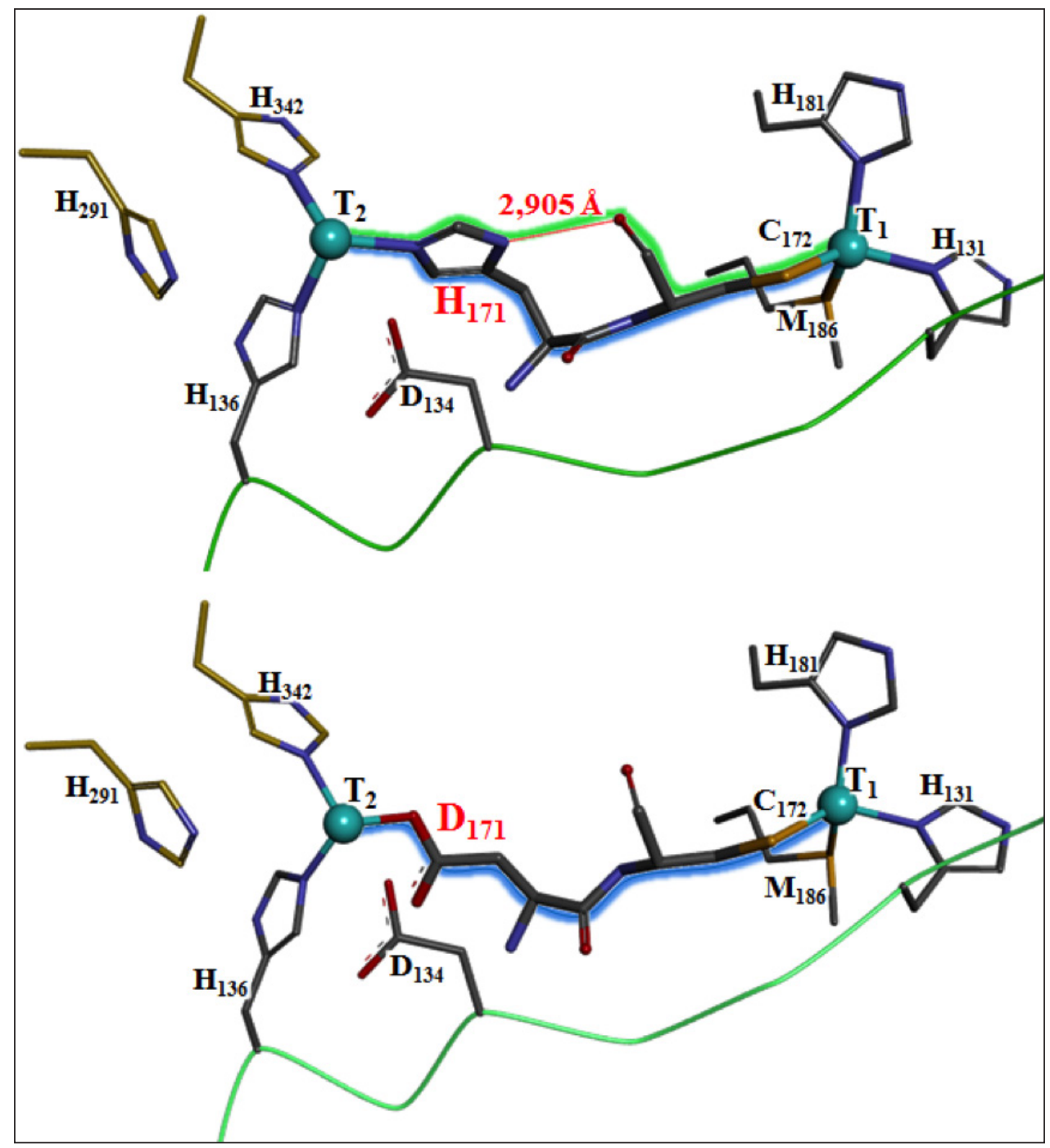

Figure 7. T1-T2 bridging pathways in wild-type $S$. meliloti NirK (top) and H171D variant (bottom) Figures were constructed using the atoms positions obtained from QM/MM calculations (Cristaldi et al., 2018). The $\mathrm{N}^{\delta 1} \mathrm{H} \ldots \mathrm{O}=\mathrm{C}$ hydrogen bond is depicted in red.

\section{X-ray crystallography and its use to unveil potential ET pathways}

The ability to get atomic details in three dimensions has demonstrated to be a booster in biological sciences and has helped to understand the chemistry that is carried out at the catalytic active site and/or at the ET pathway of an enzyme or protein complex. The increasing use of X-ray crystallography in macromolecules relies on (i) the relatively large number of synchrotron X-ray sources currently available in the world, (ii) the ability to automate crystallization conditions screening (robotics and in situ visualization platforms), (iii) the use of optimized beamlines with efficient single-photon-counting detectors, (iv) automation, (v) user-friendly data-acquisition systems, and (vi) powerful data-processing/analysis packages (Grimes et al., 2018; Muench et al., 2019).

Relevant information from NirK and Aor X-ray structures, such as the identification of putative amino acids involved in the ET mechanism and the putative regions of interaction with external ED, have been obtained in the last years (Nojiri 2017). For NirK, X-ray crystallography has provided all the structural information regarding the catalytic T2 site and its surrounding area, the ways in which T2 and T1 are connected by the His-Cys bridge, the sensing loop, and the identification of second sphere copper coordination ligands relevant in catalysis (Strange et al., 1999; Boulanger et al., 2000; Kataoka et al., 2000). All this information has been valuable in elucidating the catalytic mechanism of nitrite reduction at T2, and the T1-T2 ET process. The roles of Asp ${ }^{\mathrm{CAT}}$ and His ${ }^{\mathrm{CAT}}$ (Figure 1) as relevant players in the catalytic mechanism of NirK were based on crystallographic structures and the confirmation of their relevance achieved performing NirK variants through site directed mutagenesis. Remarkably, the identification of two Asp ${ }^{\text {CAT }}$ conformations, called proximal and gate keeper, led to suppose that this residue accomplishes an essential role in catalysis (Antonyuk 
et al., 2005; Horrell et al., 2016). The current hypothesis is that Asp ${ }^{\mathrm{CAT}}$ would sense the binding of nitrite at T2 and then, through an unknown mechanism, would trigger the ET from T1 to T2 through the Cys-His pathway (Strange et al., 1999; Cristaldi et al., 2018). Even though X-ray crystallography cannot give in fact any evidence for this ET process, it opens up distinct types of possibilities that merit to be investigated by other techniques. X-ray crystallography in conjunction with EPR has also been essential in characterizing the FeS2-FeS1-Mo ET pathway in D. gigas Aor, which is explained above under Sections 5, 6, and 7.

More recently, one particularity of X-ray crystallography, thought to be a weakness of the technique, enlarged its capability. It is well known that X-ray radiation photoreduces metal centers in several cases, though the structures so determined are thought to be those of the oxidized proteins. Particularly, the T1 centers in NirK are prone to photoreduction by X-rays during data collection (Hough et al., 2008). The T2 site of the resting-state enzyme, in contrast, remains unaffected, even at much higher X-ray doses. This fact determines that when a nitrite is bound to T2 in crystals soaked with substrate, the nitrite is converted to nitric oxide during X-ray data collection, indicating that T1-T2 ET is occurring under irradiation. Exploiting these features and using a serial crystallography approach (Horrell et al., 2016), it was possible to obtain a structural movie capturing the conversion of nitrite to nitric oxide and the subsequent return to the enzyme resting state.

Nowadays, genome mining procedures allowed identifying putative novel NirK, with X-ray crystallography being not only the key to unveil their structures but also to predict the involvement of metal cofactors in ET processes. These novel proteins emerged as a new group of NirK which are arranged in a three-domain structure containing, in addition to the two-domain core with T1 and T2 centers, an extra domain with an additional metal cofactor. X-ray crystallographic data confirmed the presence of copper cofactor C-terminus-tethering in NirK from Thermus scotoductus SA-01 (Figure 8) (Opperman et al., 2019), an extra heme c group at C-terminus in NirK from Ralstonia picketii (Antonyuk et al., 2013), an extra N-terminus cupredoxin domain in the non-self-efficient NirK from Hypomicrobium denitrificans (Nojiri et al., 2007), and an extra C-terminus heme c binding domain in NirK from Pseudoalteromonas haloplanktis (Tsuda et al., 2013). The copper extra cofactor present in H. denitrificans NirK was suggested to be involved in the intra-protein ET pathway (Deligeer et al., 2002; Yamaguchi et al., 2004), but X-ray structural data combined with mutagenesis and kinetic assays using external electron donors refuted that assumption (Nojiri et al., 2007). Even more, the functionality of $R$. picketii NirK and P. haloplanktis NirK was solely discussed based on terms of the X-ray structures (Antonyuk et al., 2013; Tsuda et al., 2013), such as intercofactor distances and nature of the chemical pathway linking the putative redox centers. However, additional experiments, as the ones explained in the sections above, are mandatory to confirm these hypotheses based only on X-ray structures.

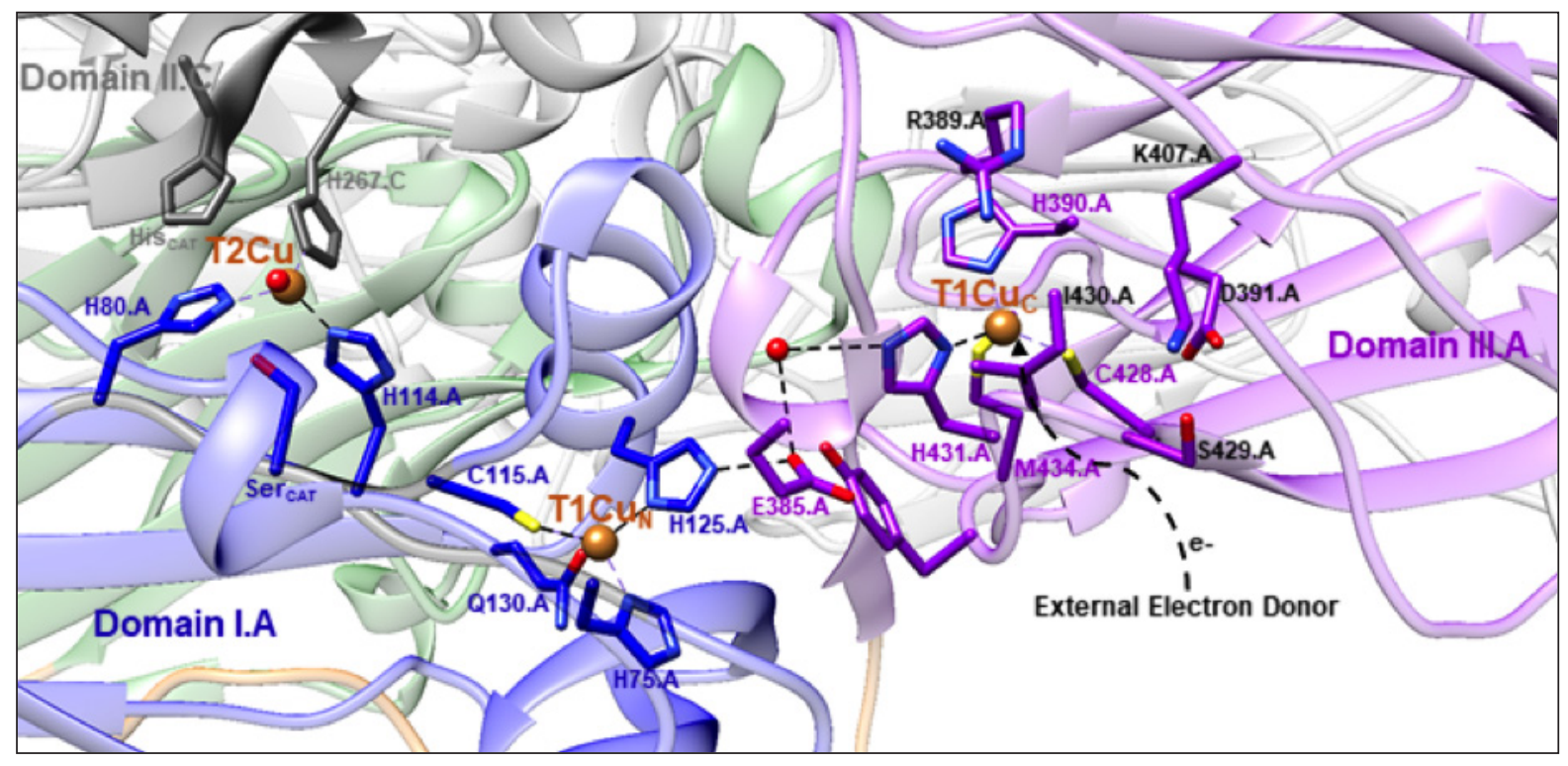

Figure 8. Metal cofactors and putative intraprotein electron transfer pathway in T. scotoductus NirK. The ET pathway


through a hydrophobic patch surrounded by charged amino acids (residues in purple), ii) from $\mathrm{T} 1 \mathrm{Cu}_{\mathrm{C}}$ to $\mathrm{T} 1 \mathrm{Cu}_{\mathrm{N}}$ through a water molecule and Glu385 residue, and iii) from $\mathrm{T}_{1 \mathrm{Cu}}$ to $\mathrm{T} 2 \mathrm{Cu}$ by either Cys-His bridge or the sensing loop. 


\section{Conclusions}

This paper summarizes the use of various experimental techniques employed in the characterization of electron transfer pathways in oxidoreductases. The examples presented have been selected to show how the combination of molecular biology, spectroscopic, electrochemical, and structural techniques can be used to characterize the chemical pathways for electron transfer process in redox enzymes and electron transfer proteins. Although the methodologies used to study these types of systems are rather different, we present in this paper a unified view of the problem to show the multidisciplinary experimental approach of the subject.

It is clear that UV-vis and EPR in conjunction with other spectroscopies not discussed here are valuable tools to study not only the geometric and electronic structure of individual paramagnetic transition metal ions, but also to evaluate $\Delta G^{\circ}$ required in all long-distance electron transfer reactions. The biggest advantage of the spectropotentiometric techniques relative to those based on voltamperometry is that, in general, with just one technique one could discriminate all the redox active species present in an electron transfer pathway. The measurement of exact values for $T_{\mathrm{DA}}$ in an independent manner is more complicated in these oxidoreductases. For those systems presenting paramagnetic redox centers coupled by isotropic exchange, it is evident that the information relative to the $T_{\mathrm{DA}}$ term provided by EPR is very valuable, as not only the ability of a chemical pathway to mediate electron transfer can be inferred, but also the order of magnitude of $T_{\mathrm{DA}}$ can be estimated. However, this "paramagnetic redox centers coupled by exchange" characteristic is not present in all electron transfer systems, and therefore additional experimental approaches have to be implemented. Among them, structural techniques can provide the starting information as potential electron transfer pathways can be envisaged, but evidently these hypothetical conclusions must be proved with other techniques. Under this context, site directed mutagenesis studies appear as one of the election techniques, since the construction of specifically designed protein variants, whose kinetic properties can be measured in comparison with those of the wild type enzymes, constitutes in our opinion a powerful method that complements structural X-ray and spectroscopic data. Evidently, the information that can be obtained with all these methodologies is in most cases either qualitative or semiquantitative at the most, as they are not oriented to measure the $k_{\mathrm{ET}}$ specifically. However, the information gathered from all the experimental techniques presented here is mandatory to fully understand at a molecular level electron transfer processes in redox enzymes.

\section{Acknowledgments}

We thank FONCyT, CONICET, and CAI+D-UNL for financial support. M.G.R. P.J.G, F.M.F., and C.D.B are members of CONICET (Argentina).

\section{References}

[1] S. L. Andrade, C. D. Brondino, M. J. Feio, I. Moura and J. J. Moura. Aldehyde oxidoreductase activity in Desulfovibrio alaskensis NCIMB 13491 EPR assignment of the proximal [2Fe-2S] cluster to the Mo site. Eur. J. Biochem., 267(7): 2054-2061, 2000.

[2] C. Andreini, I. Bertini, G. Cavallaro, G. Holliday and J. Thornton. Metal ions in biological catalysis: from enzyme databases to general principles. J Biol Inorg Chem, 13(8): 1205-1218, 2008.

[3] S. V. Antonyuk, H. Cong, R. R. Eady and S. S. Hasnain. Structures of Protein-Protein Complexes involved in electron transfer. Nature, 496(7443): 123-126, 2013.

[4] S. V. Antonyuk, R. W. Strange, G. Sawers, R. R. Eady and S. S. Hasnain. Atomic resolution structures of resting-state, substrate- and product-complexed $\mathrm{Cu}$-nitrite reductase provide insight into catalytic mechanism. Proc. Natl. Acad. Sci. U.S.A., 102(34): 12041, 2005.

[5] J. M. Berg, J. L. Tymoczko, L. Stryer and G. J. Gatto. Biochemistry. W. H. Freeman and Company, New York, USA, 2012

[6] D. R. Boer, A. Thapper, C. D. Brondino, M. J. Romao and J. J. Moura. X-ray crystal structure and EPR spectra of "arsenite-inhibited" Desulfo vibrio aldehyde dehydrogenase: a member of the xanthine oxidase family. J. Am. Chem. Soc., 126(28): 8614-8615, 2004.

[7] M. J. Boulanger, M. Kukimoto, M. Nishiyama, S. Horinouchi and M. E. P. Murphy. Catalytic Roles for Two Water Bridged Residues (Asp-98 and His-255) in the Active Site of Copper-containing Nitrite Reductase. J. Biol. Chem., 
275(31): 23957-23964, 2000.

[8] C. D. Brondino, M. G. Rivas, M. J. Romao, J. J. Moura and I. Moura. Structural and electron paramagnetic resonance (EPR) studies of mononuclear molybdenum enzymes from sulfate-reducing bacteria. Acc. Chem. Res., 39(10): 788796, 2006.

[9] R. Calvo, E. C. Abresch, R. Bittl, G. Feher, W. Hofbauer, R. A. Isaacson, W. Lubitz, M. Y. Okamura and M. L. Paddock. EPR Study of the Molecular and Electronic Structure of the Semiquinone Biradical QA-•QB-• in Photosynthetic Reaction Centers from Rhodobacter sphaeroides. J. Am. Chem. Soc., 122(30): 7327-7341, 2000.

[10] A. Cornish-Bowden. Fundamentals of enzyme kinetics. Portland, London, 2004.

[11] C. Creutz. Mixed Valence Complexes of d5-d6 Metal Centers. In Progress in Inorganic Chemistry. John Wiley and Sons, Inc, 1983.

${ }^{[12]}$ J. C. Cristaldi, M. C. Gomez, P. J. Gonzalez, F. M. Ferroni, S. D. Dalosto, A. C. Rizzi, M. G. Rivas and C. D. Brondino. Study of the Cys-His bridge electron transfer pathway in a copper-containing nitrite reductase by sitedirected mutagenesis, spectroscopic, and computational methods. Biochim. Biophys. Acta Gen. Subj., 1862(3): 752760, 2018.

[13] V. L. Davidson. Unraveling the kinetic complexity of interprotein electron transfer reactions. Biochemistry, 35(45): 14035-14039, 1996.

[14] V. L. Davidson. Chemically gated electron transfer. A means of accelerating and regulating rates of biological electron transfer. Biochemistry, 41(50): 14633-14636, 2002.

[15] V. L. Davidson. Protein control of true, gated, and coupled electron transfer reactions. Acc. Chem. Res., 41(6): 730$738,2008$.

${ }^{[16]}$ Deligeer, R. Fukunaga, K. Kataoka, K. Yamaguchi, K. Kobayashi, S. Tagawa and S. Suzuki. Spectroscopic and functional characterization of $\mathrm{Cu}$-containing nitrite reductase from Hyphomicrobium denitrificans A3151. J. Inorg. Biochem., 91(1): 132-138, 2002.

${ }^{[17]}$ F. Ferroni, J. Marangon, N. Neuman, J. Cristaldi, S. Brambilla, S. Guerrero, M. Rivas, A. Rizzi and C. Brondino. Pseudoazurin from Sinorhizobium meliloti as an electron donor to copper-containing nitrite reductase: influence of the redox partner on the reduction potentials of the enzyme copper centers. J Biol Inorg Chem, 19(6): 913-921, 2014.

${ }^{[18]}$ S. Formosinho and M. Barroso. Proton-Coupled Electron Transfer: A Carrefour of Chemical Reactivity Traditions. The Royal Society of Chemistry, Cambridge, 2012.

[19] Y. Fukuda, K. M. Tse, T. Nakane, T. Nakatsu, M. Suzuki, M. Sugahara, S. Inoue, T. Masuda, F. Yumoto, N. Matsugaki, E. Nango, K. Tono, Y. Joti, T. Kameshima, C. Song, T. Hatsui, M. Yabashi, O. Nureki, M. E. P. Murphy, T. Inoue, S. Iwata and E. Mizohata. Redox-coupled proton transfer mechanism in nitrite reductase revealed by femtosecond crystallography. Proc. Natl. Acad. Sci. U.S.A., 113(11): 2928-2933, 2016.

${ }^{[20]}$ M. Gómez, N. Neuman, S. Dalosto, P. González, J. G. Moura, A. Rizzi and C. Brondino. Isotropic Exchange Interaction between Mo and the Proximal FeS Center in the Xanthine Oxidase Family Member Aldehyde Oxidoreductase from Desulfovibrio gigas on Native and Polyalcohol Inhibited Samples: An EPR and QM/MM Study. J Biol Inorg Chem, 20(2): 233-242, 2015.

${ }^{[21]}$ P. J. González, G. I. Barrera, A. C. Rizzi, J. J. G. Moura, M. C. G. Passeggi and C. D. Brondino. EPR studies of the Mo-enzyme aldehyde oxidoreductase from Desulfovibrio gigas: An application of the Bloch-Wangsness-Redfield theory to a system containing weakly-coupled paramagnetic redox centers with different relaxation rates. J. Inorg. Biochem., 103(10): 1342-1346, 2009.

${ }^{[22]}$ J. M. Grimes, D. R. Hall, A. W. Ashton, G. Evans, R. L. Owen, A. Wagner, K. E. McAuley, F. von Delft, A. M. Orville, T. Sorensen, M. A. Walsh, H. M. Ginn and D. I. Stuart. Where is crystallography going? Acta Crystallogr. D, 74(2): 152-166, 2018. 
${ }^{[23]}$ R. G. Hadt, S. I. Gorelsky and E. I. Solomon. Anisotropic covalency contributions to superexchange pathways in type one copper active sites. J. Am. Chem. Soc., 136(42): 15034-15045, 2014.

[24] R. Hille. The Mononuclear Molybdenum Enzymes. Chem. Rev., 96(7): 2757-2816, 1996.

[25] J. J. Hopfield. Electron transfer between biological molecules by thermally activated tunneling. Proc. Natl. Acad. Sci. $U S A, 71(9): 3640-3644,1974$.

[26] S. Horrell, S. V. Antonyuk, R. R. Eady, S. S. Hasnain, M. A. Hough and R. W. Strange. Serial crystallography captures enzyme catalysis in copper nitrite reductase at atomic resolution from one crystal. IUCrJ, 3(4): 271-281, 2016.

${ }^{[27]}$ M. A. Hough, R. R. Eady and S. S. Hasnain. Identification of the Proton Channel to the Active Site Type 2 Cu Center of Nitrite Reductase: Structural and Enzymatic Properties of the His254Phe and Asn90Ser Mutants. Biochemistry, 47(51): 13547-13553, 2008.

${ }^{[28]}$ M. K. Johnson. Iron-sulfur proteins: new roles for old clusters. Curr. Opin. Chem. Biol., 2(2): 173-181, 1998.

${ }^{[29]}$ K. Kataoka, H. Furusawa, K. Takagi, K. Yamaguchi and S. Suzuki. Functional Analysis of Conserved Aspartate and Histidine Residues Located Around the Type 2 Copper Site of Copper-Containing nitrite reductase. J. Biochem., 127(2): 345-350, 2000.

${ }^{[30]}$ L. Krippahl, P. N. Palma, I. Moura and J. J. G. Moura. Modelling the electron-transfer complex between aldehyde oxidoreductase and flavodoxin. Eur. J. Inorg. Chem.(19): 3835-3840, 2006.

[31] C. Leger and P. Bertrand. Direct electrochemistry of redox enzymes as a tool for mechanistic studies. Chem. Rev., 108(7): 2379-2438, 2008.

${ }^{[32]}$ R. A. Marcus and N. Sutin. Electron transfers in chemistry and biology. Biochim. Biophys. Acta- Reviews on Bioenergetics, 811(3): 265-322, 1985.

33] C. C. Moser, J. L. Anderson and P. L. Dutton. Guidelines for tunneling in enzymes. Biochim. Biophys. Acta, 1797(9): $1573-1586,2010$.

${ }^{[34]}$ J. J. Moura, A. V. Xavier, R. Cammack, D. O. Hall, M. Bruschi and J. Le Gall. Oxidation-reduction studies of the Mo(2Fe-2S) protein from Desulfovibrio gigas. Biochem. J., 173(2): 419-425, 1978.

[35] S. P. Muench, S. V. Antonyuk and S. S. Hasnain. The expanding toolkit for structural biology: synchrotrons, X-ray lasers and cryoEM. IUCrJ, 6(2): 167-177, 2019.

${ }^{[36]}$ M. Nojiri. Structure and Function of Copper Nitrite Reductase. In Metalloenzymes in Denitrification: Applications and Environmental Impacts. The Royal Society of Chemistry, Cambridge, 2017.

${ }^{[37]}$ M. Nojiri, Y. Xie, T. Inoue, T. Yamamoto, H. Matsumura, K. Kataoka, Deligeer, K. Yamaguchi, Y. Kai and S. Suzuki. Structure and function of a hexameric copper-containing nitrite reductase. Proc. Natl. Acad. Sci. U.S.A., 104(11): 4315-4320, 2007.

${ }^{[38]}$ K. Olesen, A. Veselov, Y. Zhao, Y. Wang, B. Danner, C. P. Scholes and J. P. Shapleigh. Spectroscopic, Kinetic, and Electrochemical Characterization of Heterologously Expressed Wild-Type and Mutant Forms of Copper-Containing Nitrite Reductase from Rhodobacter sphaeroides 2.4.3†. Biochemistry, 37(17): 6086-6094, 1998.

${ }^{[39]}$ D. J. Opperman, D. H. Murgida, S. D. Dalosto, C. D. Brondino and F. M. Ferroni. A three-domain copper-nitrite reductase with a unique sensing loop. IUCrJ, 6(2): 248-258, 2019.

${ }^{[40]}$ C. C. Page, C. C. Moser, X. Chen and P. L. Dutton. Natural engineering principles of electron tunnelling in biological oxidation-reduction. Nature, 402(6757): 47-52, 1999.

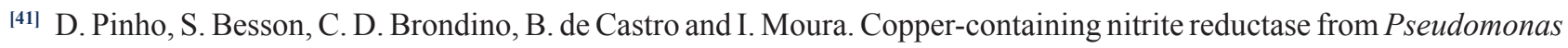
chlororaphis DSM 50135. Eur. J. Biochem., 271(12): 2361-2369, 2004. 
${ }^{[42]}$ J. M. Rebelo, J. M. Dias, R. Huber, J. J. Moura and M. J. Romao. Structure refinement of the aldehyde oxidoreductase from Desulfovibrio gigas (MOP) at 1.28 A. J Biol Inorg Chem, 6(8): 791-800, 2001.

${ }^{[43]}$ A. C. Rizzi, P. J. Gonzalez, N. Neuman and C. D. Brondino. EPR as a Tool for Study of Isolated and Coupled Paramagnetic Centers in Coordination Compounds and Macromolecules of Biological Interest. Eur. J. Inorg. Chem., 2016(2): 192-207, 2016.

${ }^{[44]}$ M. J. Romao, M. Archer, I. Moura, J. J. Moura, J. LeGall, R. Engh, M. Schneider, P. Hof and R. Huber. Crystal structure of the xanthine oxidase-related aldehyde oxido-reductase from D. gigas. Science, 270(5239): 1170-1176, 1995.

${ }^{[45]}$ T. Santos-Silva, F. Ferroni, A. Thapper, J. Marangon, P. J. González, A. C. Rizzi, I. Moura, J. J. G. Moura, M. J. Romão and C. D. Brondino. Kinetic, structural, and EPR studies reveal that aldehyde oxidoreductase from Desulfovibrio gigas does not need a sulfido ligand for catalysis and give evidence for a direct Mo-C interaction in a biological system. J. Am. Chem. Soc., 131(23): 7990-7998, 2009.

${ }^{[46]}$ J. J. R. F. d. Silva and R. J. P. Williams. The biological chemistry of the elements : the inorganic chemistry of life. Clarendon Press, Oxford, 1991.

${ }^{[47]}$ R. W. Strange, L. M. Murphy, F. E. Dodd, Z. H. L. Abraham, R. R. Eady, B. E. Smith and S. S. Hasnain. Structural and kinetic evidence for an ordered mechanism of copper nitrite reductase. J. Mol. Biol. , 287(5): 1001-1009, 1999.

${ }^{[48]}$ S. Suzuki, T. Kohzuma, Deligeer, K. Yamaguchi, N. Nakamura, S. Shidara, K. Kobayashi and S. Tagawa. Pulse Radiolysis Studies on Nitrite Reductase from Achromobacter cycloclastes IAM 1013: Evidence for Intramolecular Electron Transfer from Type $1 \mathrm{Cu}$ to Type $2 \mathrm{Cu}$. J. Am. Chem. Soc., 116(24): 11145-11146, 1994.

${ }^{[49]}$ A. Tsuda, R. Ishikawa, H. Koteishi, K. Tange, Y. Fukuda, K. Kobayashi, T. Inoue and M. Nojiri. Structural and mechanistic insights into the electron flow through protein for cytochrome c-tethering copper nitrite reductase. $J$. Biochem., 154(1): 51-60, 2013.

${ }^{[50]}$ K. Yamaguchi, K. Kataoka, M. Kobayashi, K. Itoh, A. Fukui and S. Suzuki. Characterization of Two Type $1 \mathrm{Cu}$ Sites of Hyphomicrobium denitrificans Nitrite Reductase: A New Class of Copper-Containing Nitrite Reductases. Biochemistry, 43(44): 14180-14188, 2004.

\section{Bios}

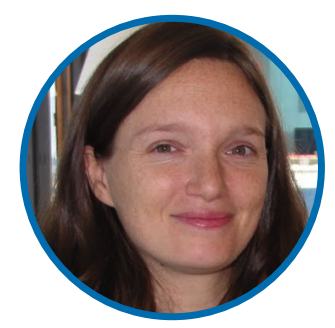

\section{María Gabriela Rivas}

María Gabriela Rivas received her B.Sc. degree in Biotechnology from Universidad Nacional del Litoral (Argentina), followed by a Ph.D. in Biophysical Chemistry from the Department of Chemistry at the Faculdade de Ciências e Tecnologia of Universidade Nova de Lisboa (Portugal). From 2007 to 2009, she was a postdoctoral fellow at REQUIMTE (Portugal) and CNRS (France). Since 2013, she is a research scientist for CONICET and a professor at the Department of Physics of the School of Biochemistry and Biological Sciences of Universidad Nacional del Litoral (Argentina). Her main research interest is the study of molecular mechanisms for Mo and $\mathrm{W}$-uptake in bacteria.

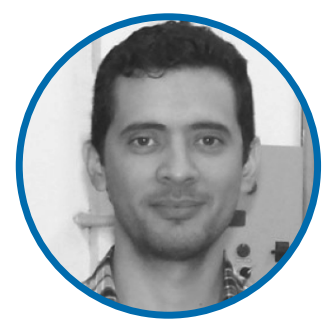

\section{Pablo J. González}

Pablo J. González received his diploma in Biotechnology from Universidad Nacional del Litoral in 2002. In 2006, he obtained his Ph.D. degree in Biophysical Chemistry from the Department of Chemistry at the Faculdade de Ciências e Tecnologia of Universidade Nova de Lisboa (Portugal). He had postdoctoral training in biophysical techniques applied to the study of metalloproteins before joining as a research scientist for REQUIMTE at FCTUNL in Portugal. He is currently a professor at the Department of Physics and a research scientist for CONICET at the School of Biochemistry and Biological Sciences of Universidad Nacional del Litoral, Argentina. 


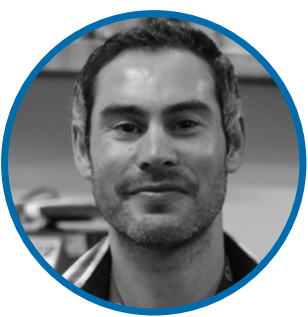

\section{Felix M Ferroni}

Felix M Ferroni was born in San Pedro (Bs. As.), Argentina, in 1979. He obtained his degree in Biotechnology with highest honors in 2005, followed by a $\mathrm{PhD}$ in Biological Sciences in 2010, both at Universidad Nacional del Litoral, Argentina. He continued his career with a postdoc at CONICET (2010-2012) and a postdoc in biocatalysis and protein crystallography (20132015) at University of The Free State (South Africa). $\mathrm{He}$ is currently an Assistant Researcher for CONICET; his main research interest is the study of enzymes with cupredoxin-domains from extremophiles and soil bacteria with biocatalytic, crystallographic, electrochemical and biosesing purposes.

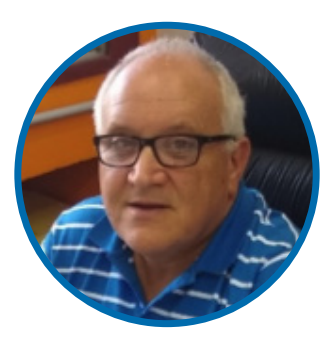

\section{Alberto C. Rizzi}

Alberto C. Rizzi received B.Sc. and Ph.D. degrees from Universidad Nacional del Litoral (Argentina) and was a postdoctoral fellow at Max Planck Institute for Bioinorganic
Chemistry (Germany). He is now associate professor at the Department of Physics of the School of Biochemistry and Biological Sciences at Universidad Nacional del Litoral. His research interests include the role of transition metals ions in biology and the study of bioinorganic systems as models for complex biological compounds using Electronic Paramagnetic Resonance (EPR) and magnetic measurements.

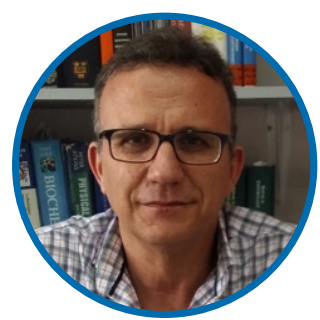

\section{Carlos D. Brondino}

Carlos D. Brondino received his B.Sc. and Ph.D. degrees from Universidad Nacional del Litoral (Argentina). He was a postdoctoral and research fellow at the Department of Chemistry of Universidade Nova de Lisboa, Portugal. He is now a full professor, head of Department, and a research fellow for CONICET at the Department of Physics of the School of Biochemistry and Biological Sciences of Universidad Nacional del Litoral. His main research interests include the reaction mechanism of metalloenzymes involved in the biological cycles of nitrogen and sulfur, and the development of EPR-based methodologies to characterize the magnetic properties of paramagnetic systems of biological interest. 